\title{
La carta al editor en la publicación científica. Consideraciones para su elaboración
}

\author{
The letter to the editor in the scientific publication. \\ Considerations for its preparation \\ A carta ao editor na publicação científica. \\ Considerações para sua elaboração \\ Yuri Castro-Rodríguez' (D) 0000-0002-9587-520X
}

\section{Resumen}

Una carta al editor es un tipo de publicación científica que se clasifica como "comunicación corta” y que permite a los lectores interactuar con los autores ya sea a través de opiniones, críticas, aportes, ideas, hipótesis y nuevos datos. En el presente artículo se realizó una revisión bibliográfica sobre las principales consideraciones para la redacción y elaboración de una carta al editor. Se exploró los artículos de las bases de datos PubMed, Scopus y SciELO desde el año 2010 (enero) hasta la actualidad (diciembre del 2020). La carta al editor como publicación científica presenta consideraciones para su redacción y construcción que pueden hacer referencia a un artículo publicado en la revista, así como a una temática coyuntural o aporte del lector. Permite confrontar las ideas publicadas por los investigadores con una comunidad académica. Desde un enfoque pedagógico permite a un estudiante e investigador novel iniciarse en el mundo de la crítica y redacción científica; asimismo conocer el proceso editorial de envío y publicación de artículos científicos.

Palabras clave: Conocimiento; Carta, Publicaciones Científicas y Técnicas; Artículo de revista.

Universidad Nacional Mayor de San Marcos. Lima, Perú. yuricastro_16@hotmail.com

Fecha de recibido: 6/12/2020 - Fecha de aceptado: 22/2/2021 


\section{Abstract}

A letter to the editor is a type of scientific publication that is classified as "short communication" and that allows readers to interact with authors through opinions, criticisms, contributions, ideas, hypotheses and new data. In the present article a bibliographic review was carried out on the main considerations for the writing and elaboration of a letter to the editor. If you have explored the articles of the PubMed, Scopus and SciELO databases since the year 2010 (January) up to the present time (December 2020) The letter to the editor as a scientific publication presents considerations for its writing and construction that may refer to an article published in the magazine, as well as the conjectural theme the contribution of the lector. It allows confronting the ideas published by the researchers with an academic community. From a pedagogical approach, it allows a novel student and researcher to begin in the world of criticism and scientific writing; likewise knows the editorial process of sending and publishing scientific articles.

Keywords: Knowledge; Charter, Scientific and Technical Publications; Magazine article.

\section{Introducción}

La comunicación científica incluye a la publicación de artículos científicos, sean estos: originales, revisiones, ensayos, notas, casos clínicos, etc. La publicación no es la última etapa del proceso de investigación científica, sino que es el inicio de un nuevo proceso donde interactúan lectores, autores y comunidad. Esta interacción forma parte de un nuevo discurso científico, que puede darse de muchas formas: entre colegas, en reuniones científicas, durante

\section{Resumo}

Una carta al editor é um tipo de publicação científica que se classifica como "comunicación corta" e que permite que os leitores interajam com os autores e a través de opiniôes, críticas, aportes, ideias, hipóteses e novos dados. En el presente artigo se realiza uma revisión bibliográfica sobre las principales consideraciones para la redacción y elaboración de una carta al editor. Veja os artigos das bases de dados PubMed, Scopus y SciELO desde o ano de 2010 (enero) hasta la atualidad (diciembre del 2020). La carta al editor como publicación científica apresenta consideraciones para su redacción y construcción that pueden hacer referencia a un artículo publicado en la revista, así como a una temática coyuntural o aporte del leitor. Permite confrontar as ideias publicadas por los pesquisadores com uma comunidade acadêmica. Desde um enfoque pedagógico permite um estudo e investigador de um romance iniciarse no mundo da crítica e redacción científica; asimismo conocer el proceso editorial de envío y publicación de artículos científicos.

Palavras-chave: Conocimiento; Carta, Publicaciones Científicas y Técnicas; Artículo de revista.

la revisión por pares y a través de otras publicaciones denominadas "cartas al editor".

Una carta al editor es un tipo de artículo estructuralmente "sencillo" (por lo menos más sencillo que los demás tipos de artículos), que permite favorecer el discurso científico para interpretar estudios y orientar investigaciones futuras ${ }^{(1)}$. Esta interacción entre autores y lectores no es reciente y comienza a surgir en el siglo XV cuando científicos de toda Europa intercambiaron ideas y desafiaron el pensamiento de los demás. Esto surgió debido a que en una revista, 
los artículos publicados se revisan y seleccionan meticulosamente, sin embargo algunos errores pueden haberse pasados ya sea por los revisores o por el comité editorial. En estas circunstancias, los lectores pueden enviar una carta al editor expresando sus comentarios sobre alguna publicación de la revista.

Una carta al editor es un medio de comunicación breve entre el autor de un artículo y el lector de una revista ${ }^{(2)}$. Aborda diversos temas, desde comentarios sobre temas locales, estatales, nacionales y actualidad internacional, así como respuestas a opiniones, historias, hallazgos y datos publicados en una revista científica o en un periódico ${ }^{(3)}$. En una revista científica, estas cartas no se consideran como artículos de investigación, sino comunicaciones que revisan otros artículos y ofrecen sugerencias, formas alternativas de hacer las cosas, borrar conceptos y señalar cosas que pueden haber pasado por alto ya sea por los autores o los revisores ${ }^{(4)}$. La literatura muestra que la carta al editor sirve como medida de control para los investigadores pues muchas veces aportan aspectos que los autores pasaron por alto; sin embargo también se puede utilizar para dañar la reputación de un investigador ${ }^{(5)}$.

El Comité Internacional de Editores de Revistas Biomédicas recomienda la publicación de estas cartas junto con sus respuestas. La ausencia de una sección como ella niega a los lectores la posibilidad de responder a los artículos publicados ${ }^{(6)}$. En las revistas impresas muchas veces existe un espacio limitado para la publicación, por lo que las cartas al editor se exigen que sean breves (por ejemplo, tener una extensión entre 100 a 200 palabras). En las revistas electrónicas, también se suele exigir su concisión (entre 250 a 500 palabras), por lo que escribir una carta al editor se convierte en un ejercicio útil para crear un argumento sucinto pero persuasivo ${ }^{(3)}$.

La mayoría de periódicos (no necesariamente revistas científicas) también presenta una sección de cartas al editor donde la gente puede escribir y compartir sus puntos de vista respecto a lo que publica el periódico. Esta característica se comparte con los artículos científicos, por lo que el lector debe redactar una carta y enviarla al periódico o la revista y esperar a que sea aprobada o rechazada. Si bien no hay garantías de su publicación, una carta bien redactada, actual e interesante tiene más probabilidades que el editor la acepte para su publicación.

Las cartas al editor deben tener una razón para ser redactadas, asimismo deben transmitir su mensaje en un modo corto y breve con puntos claramente definidos. Por lo común revisan aspectos desfavorables de un artículo, lo que les permite ser un mecanismo de control que facilita el progreso después de la publicación de un artículo. Sus características, importancia, desventajas y consideraciones para redactarlas presentan ciertas peculiaridades, por tal motivo, en el presente artículo se sintetizan las principales consideraciones a tener en cuenta cuando se pretenda redactar una carta al editor.

\section{Método}

Se realizó una revisión documental, donde se indagó la información publicada sobre la "carta al editor" desde la perspectiva de la publicación científica. La búsqueda de fuentes de información se realizó entre los meses de marzo a abril del 2020 e incluyó las bases de datos: SciELO (Scientific Electronic Library Online), MEDLINE (Pubmed), ScienceDirect así como el buscador Google académico. En cada base de datos se realizó una búsqueda libre de la información con un límite de 10 años de antigüedad de las fuentes de información para analizar fuentes antiguas como actuales; ubicadas las fuentes se procedió a revisar sus referencias bibliográficas para obtener mayor información. Se utilizaron los términos clave en español: "Carta al editor", "Artículos científicos", "Publicación científica" y "Redacción científica”. Los términos en inglés incluyeron: "Letter to the editor", "Scientific papers", "Scientific publication" y "Scientific redaction". 
Ubicadas las fuentes de información se analizaron según las siguientes categorías: definición de una carta al editor, importancia, desventajas y consideraciones para su redacción. El rastreo ubicó 28 fuentes de información, todas ellas artículos científicos.

\section{Desarrollo}

\section{Tipos de cartas al editor}

Las cartas al editor son un tipo de artículo científico que publica una revista. Es decisión de un comité editorial la cantidad de cartas publicadas por número de la revista, asimismo, es decisión del comité si estas cartas serán sometidas a un proceso de revisión por pares y si estas cartas tendrán una réplica por parte del editor o los autores. Pueden clasificarse de diversas formas, las principales son: cartas de comentario y cartas de observación ${ }^{(7)}$, también existen las cartas comunes y no comunes ${ }^{(4)}$ así como cartas relacionadas al tipo de publicación con la que se relaciona: cartas de reportes de caso, cartas de artículos originales, cartas de opinión ${ }^{(8)}$.

Las cartas de observación son aquellas que presentan una obra original, asemejan a un artículo original pero son más concisos; se diseñan cuando se desea comunicar un resultado de forma rápida ${ }^{(9)}$. Las cartas del tipo "comentario" son las más comunes y constituyen una crítica a las publicaciones relacionadas con la revista; su periodo de revisión es más lento (entre 3 a 6 meses, dependiendo de la revista) y suele estar relacionada a los últimos artículos publicados por la revista. En ambos tipos se evidencian posturas (a favor o en contra) del autor de la carta hacia algún artículo o tema publicado por la revista, esta postura muchas veces debe ser respaldada con fuentes de información, por lo que una carta suele presentar referencias bibliográficas.

Las cartas también pueden clasificarse como aquellas que hacen mención a artículos recientemente publicados (es recomendable los dos últimos números de la revista) ${ }^{(10)}$ y aquellos re- feridas a publicaciones antiguas de la revista ${ }^{(11)}$. Las primeras son las más comunes y se enfocan en: identificar errores y realizar correcciones, plantear teorías alternativas, agregar información importante que no fue tomada en cuenta en el artículo original, ofrecer evidencia adicional y mostrar ideas que contrasten con la idea principal del artículo original.

\section{Importancia de una carta al editor}

Las cartas al editor son un medio rápido y eficaz de comunicar problemas conocidos, poco conocidos o que no fueron tomados en cuenta por los investigadores. Se pueden utilizar para corregir y aclarar hechos en una noticia, artículo, editorial o artículo de opinión; también pueden oponerse o apoyar las acciones de una institución, dirigir la atención hacia un problema, estimular a los editores a cubrir un tema que se está pasando por alto e instar a los lectores para apoyar su causa. Si múltiples cartas se publican sobre un tema, estas pueden agregar energía y urgencia al llamado a una mayor atención al problema.

Las cartas permiten: desarrollar, discutir e intercambiar ideas, desafiar o apoyar ideas que hayan pasado por la revisión por pares, corregir errores e iniciar el diálogo entre investigadores y lectores. Ayudan a plantear puntos que no se han cubierto en un estudio así como proporcionar información adicional para respaldar el trabajo (12). Las cartas al editor, al igual que las otras herramientas de difusión de los medios, educan a los lectores sobre un tema, afectan la opinión pública e influyen en las posiciones de los lectores y la toma de decisiones ${ }^{(13)}$. No deben ser utilizadas para evitar la revisión por pares o la creación de una gran lista de publicaciones en el currículo de un autor.

Su importancia radica en que muchas de ellas detectan defectos metodológicos o de interpretación de un estudio, a veces comunican hallazgos de otros estudios que no fueron considerados por la publicación original, en el campo biomédico permiten comunica un hallazgo 
clínicos o experimentales no descritos aún. Comunican observaciones y hallazgos que por su extensión e importancia no se adaptan al formato de un artículo original (14-16). También permiten desarrollar opiniones, ideas, metodologías novedosas e hipótesis, y presentarlas a la comunidad científica ${ }^{(15)}$.

El objetivo de una carta en respuesta a un estudio publicado debe ser apoyar o criticar los métodos, análisis o resultado del estudio. Del mismo modo, la carta al editor tiene un doble papel en la literatura. Puede servir como herramienta correctiva y también para difundir y compartir conocimientos y experiencias ${ }^{(5)}$. Cuando la carta tiene el propósito de criticar un artículo, entonces debe ser breve y solo se deben hacer críticas basadas en evidencia utilizando referencias de un trabajo publicado previamente para justificar la crítica ${ }^{(12,17) .}$

Ayudan a encontrar errores que se pasaron por alto durante la revisión antes de la publicación. Esto ayuda a mantener la calidad de las revistas. Algunas cartas también pueden otorgar opiniones sobre el estilo y las políticas editoriales de la revista, esto ayuda a que el editor y su comité valoren sus prácticas y la calidad editorial. Aquí la función de un "segundo punto de vista" que tienen las cartas al editor.

Desde el punto de vista pedagógico, las cartas al editor sirven como punto de iniciación en el mundo de la investigación y de la publicación en investigadores jóvenes y estudiantes. Ello gracias a su estructura poco compleja; lo que les permite insertarse a la actividad científica además de reforzar capacidades de redacción, lectura crítica, entre otros ${ }^{(7,18)}$. De esta forma son una estrategia de formación del recurso humano para la investigación. Al estudiante le permite demostrar que es capaz de leer y criticar un artículo científico así como generar nuevas preguntas de investigación y redactar de forma coherente. Permiten acercar a los estudiantes al trabajo de los investigadores y autores de artículos científicos ${ }^{(19,20)}$; su función pedagógica permite que un estudiante se familiarice con el proceso de envío de artículos, se relacione con un editor y protocolos de una revista, estas experiencias le servirán para redactar artículos de mayor complejidad.

\section{Desventajas de una carta al editor}

Las cartas al editor pueden redactarse con la finalidad de desprestigiar a investigadores, pueden tener abusos en manos de quienes apoyan falsamente a favor o critican a un autor. Por lo que se recomienda a los autores de cartas evitar esta confrontación o intento de desprestigio a otro autor.

Algunas revistas cobran una cierta cantidad de dinero por la publicación de sus artículos. Esto a veces puede convertirse en un obstáculo para que los lectores puedan remitir sus cartas al editor, envíen sus comentarios o puntos de vista. Además, muchas revistas solo reciben cartas enviadas dentro de un período de tiempo prescrito, por lo que este límite obstaculiza el envío de cartas ${ }^{(5)}$.

Dentro de las desventajas que tiene una carta al editor es que muchas de ellas no guardan relación con los artículos o las temáticas de una revista, algunas de ellas repiten información ya conocida o cubierta por la fuente origina, su mensaje no es claro, a veces se exagera con la descripción y detalle, no aportan información nueva o útil, se exceden en tablas o figuras, utilizan referencias con la finalidad de aumentar la citación e impacto del autor, otorgan opiniones sesgadas ${ }^{(17)}$ y son utilizadas para aumentar la producción científica de un autor, autores, universidades y países.

\section{Consideraciones para redactar una carta al editor}

Una carta no suele tener más de 500 palabras (aunque esto dependerá de las directrices de la revista) y está dirigido al editor de un periódico o revista. Es recomendable que trate de un tema actual o algo controvertido. Si desea iniciar una discusión sobre un tema en particular, o responder a una carta o artículo en su periódico, 
mencione qué artículo está respondiendo y la fecha en que fue publicado, para que los lectores y el editor sepan lo que se está escribiendo.

Si no está seguro de cómo empezar, busque las cartas al editor publicadas con anterioridad en la revista, esto puede ayudarlo a obtener ideas y visualizar el estilo que sigue la revista. Esta búsqueda también permite identificar algunas preguntas como: ¿Sobre qué temas se está publicando?
¿Cuáles son los temas que más se publican? ¿Qué se dice sobre los problemas? ¿Qué opinan de ese problema? ¿Coincide con lo ya dicho o tiene un nuevo aporte? Cuando tenga claro el problema a enfocar, revise otros artículos que servirán de sustento para la redacción de la carta. Hacer referencia a un artículo o columna publicados recientemente aumentará las posibilidades de que su carta se publique (Tabla 1).

\section{Tabla 1. Consideraciones para la redacción de una carta al editor}

\begin{tabular}{|c|c|}
\hline Recomendación & Consideración \\
\hline $\begin{array}{l}\text { Investigue las directrices para los } \\
\text { autores }\end{array}$ & $\begin{array}{l}\text { La extensión de una carta al editor varía de revista en revista, son comunes las extensiones entre } \\
250 \text { a } 500 \text { palabras. Considere que las revistas aceptan los envíos ya sea a través de una plataforma } \\
0 \text { a través de un correo electrónico. Verifique que la revista acepta cartas al editor y si se tiene } \\
\text { alguna consideración del tipo de carta que se acepta. Revise las cartas ya publicadas para conocer } \\
\text { su extensión, su temática y su estilo. }\end{array}$ \\
\hline Redacte un título llamativo & $\begin{array}{l}\text { El título debe reflejar la temática de la carta, debe ser breve (consulte la cantidad de palabras que } \\
\text { acepta la revista en cuanto al título). Por lo común las cartas al editor pueden presentar títulos } \\
\text { exclamativos, interrogativos, o con algún juego de palabras. }\end{array}$ \\
\hline $\begin{array}{l}\text { En lo posible escoja un tema } \\
\text { coyuntural }\end{array}$ & $\begin{array}{l}\text { El lector se interesará más por los temas que afectan a su vida y comunidad, sean estos económi- } \\
\text { cos, políticos o sociales. Otorgue hechos y datos que aumenten la relevancia del problema. }\end{array}$ \\
\hline Hágalo relevante & Deje claro que su tema es relevante e importante y argumente esa importancia. \\
\hline No asuma nada & $\begin{array}{l}\text { No asuma que sus lectores están informados sobre el tema, otorgue un preámbulo y trasfondo in- } \\
\text { formativo. Incluya citas y fuentes que demuestren que se ha informado del tema. No tenga miedo } \\
\text { de expresar su opinión con claridad; sin embargo se recomienda evitar el lenguaje emotivo. }\end{array}$ \\
\hline Sea coherente y cohesivo & $\begin{array}{l}\text { Plantee desde el primer párrafo su posición. Construya párrafos cortos. Apoye su posición con } \\
\text { hechos sucintos. De preferencia que un párrafo plantee solo una idea. Evite oraciones largas pues } \\
\text { hacen que el lector pierda interés. Plantee párrafos cohesionados, enlazados según la idea que } \\
\text { se esté planteando. Mantenga su escritura activa y persuasiva para que el público quiera seguir } \\
\text { leyendo. }\end{array}$ \\
\hline Sea respetuoso y ético & $\begin{array}{l}\text { Evite expresiones que dañen la honra de otra persona, critique a la idea } 0 \text { al método. Utilice un } \\
\text { lenguaje estándar o superestándar, cuidando la ortografía y gramática. No personifique sus críticas. }\end{array}$ \\
\hline Identifíquese & $\begin{array}{l}\text { Otorgue sus datos personales y filiación, de esta forma sus lectores y otros autores sabrán quién } 0 \\
\text { quiénes los están criticando o comentando. }\end{array}$ \\
\hline Sea oportuno & $\begin{array}{l}\text { Las cartas al editor suelen ser una respuesta o una reacción a una } \\
\text { artículo que apareció en la revista en un momento especíico. La mayoría de revistas aceptan cartas } \\
\text { dirigidas a los artículos publicados en los últimos números. }\end{array}$ \\
\hline Use fuentes de información & $\begin{array}{l}\text { Si la carta va a criticar algún aspecto metodológico, dato o plantear una hipótesis; es recomendable } \\
\text { que los argumentos cuenten con un respaldo teórico. }\end{array}$ \\
\hline Use su autoría y filiación & $\begin{array}{l}\text { Si usted es una autoridad en su campo de estudio considere firmar con ese título pues permitirá } \\
\text { que la carta tenga un mayor respaldo e impacto. }\end{array}$ \\
\hline Empiece fuerte & $\begin{array}{l}\text { El primer párrafo debe captar la atención del lector y editor ya sea indicando la problemática, su } \\
\text { postura o una frase de reflexión. }\end{array}$ \\
\hline Informe soluciones & $\begin{array}{l}\text { Si se trata de un tema problemático otorgue propuestas de solución e intente hacer un llamamien- } \\
\text { to a la acción. }\end{array}$ \\
\hline
\end{tabular}

Fuente: Elaboración propia 
Para la revistas biomédicas muchas de ellas recomiendan que las cartas y sus respuestas sean breves (entre dos a tres párrafos como máximo) además de presentar como máximo cinco referencias bibliográficas; aunque esto dependerá de las exigencias de la revista. En lo posible evitar presentar figuras y tablas (a menos que sea indispensable). El título debe ser informativo, mientras que el contenido de presentar un contenido progresivo para finalizar con un comentario final a modo de conclusión ${ }^{(21)}$ (Fig. 1).

Fig. 1. Esquema general de una carta al editor.

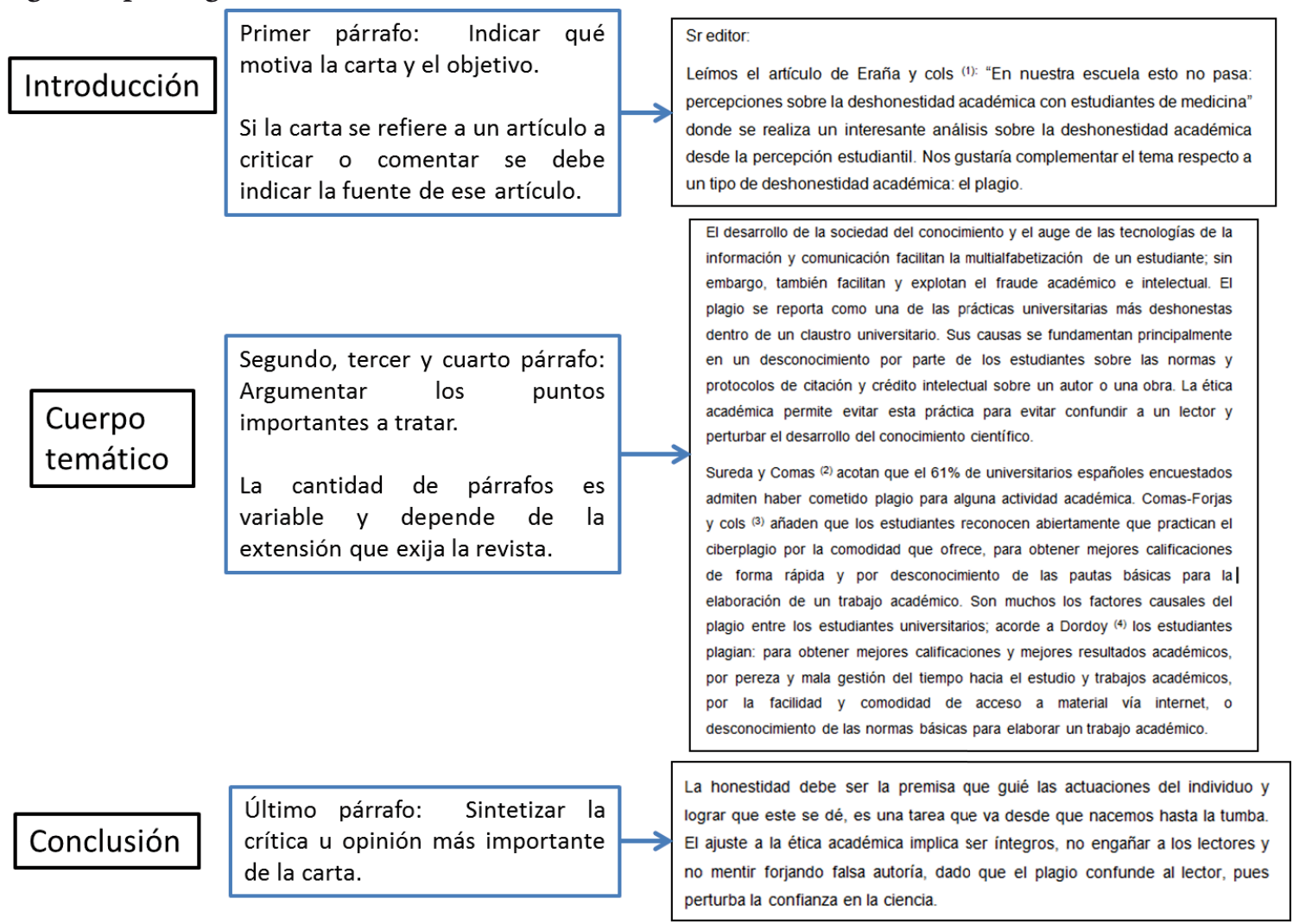

\section{Discusión}

Las investigaciones en publicación son propensas a errores, mal uso de estadísticas, citas selectivas de trabajos publicados, citas erróneas de referencias, a menudo realizadas sin saberlo, lo que puede escapar a la revisión por pares antes de que se publique el artículo ${ }^{(5)}$. Solo después de la publicación, el artículo está más abierto a la revisión crítica. Las cartas al editor permiten intercambiar comunicación con los editores de las revistas y ser un elemento que permite veri- ficar la revisión por pares de las mismas ${ }^{(22)}$. Los editores aprecian esas cartas, no solo porque prueban que alguien está leyendo la revista y se lo toman en serio sino también porque normalmente aportan críticas reflexivas para el trabajo editorial.

En la presente revisión se describieron las características, funciones, tipos y estructura de una carta al editor. Se encontró que las revistas científicas utilizan distintos tipo de cartas: algunas cartas describen casos interesantes, otras son 
opiniones expresadas por los lectores. Además, muchos editores publican estudios cortos que no califican para un artículo original completo, en forma de cartas. También existen cartas que plantean una nueva hipótesis, describen un novedoso evento adverso o un punto relevante para la práctica clínica, asimismo se encuentran las respuestas o comentarios a los artículos publicados en la revista. Por lo común las cartas hacen mención a los artículos recientemente publicados por una revista; sin embargo, las revistas pueden exigirlas para tratar puntos temáticos de interés en una comunidad. Una carta al editor también puede aportar información nueva y de importancia científica, independiente de anteriores publicaciones; publicando por ejemplo reportes cortos, o trabajos de investigación cuyo contenido justifique el ser compartido, pero cuya extensión no permita una publicación como un artículo original completo ${ }^{(22,23)}$. Para una revista científica, incluir el segmento de cartas al editor permite otorgar a los lectores la oportunidad de comunicarse con el equipo editor y con los autores de los artículos. Proporciona un foro para preguntar, comentar y criticar artículos o solicitar aclaraciones. Crea un ambiente de comunicación entre autores y lectores sirviendo como un foro de dialogo y debate ante la publicación de nuevos hallazgos. Dentro de ello, las observaciones metodológicas o estadísticas, interpretación de los resultados y definiciones operacionales. Así también, permiten comunicar algún hallazgo clínico o experimental previamente no descrito; o incluso emitir opinión sobre aspectos de la política editorial de una revista, entre otros. Además permiten que el autor brinde una explicación (réplica) en relación con los aspectos sobre los que ha sido cuestionado; siendo esto, una de las recomendaciones del Comité Internacional de Editores de Revistas Médicas ${ }^{(24,25)}$.

Cabe indicar que cuando se redacta una carta al editor se debe pensar en el lector y no en el editor. Muchas veces las cartas son dirigidas al editor con la finalidad de que él "haga algo" o tome en consideración la postura de los autores. Esta consideración debe ser evitada por los autores pues se trata de que la carta manifieste algún tipo de crítica reflexivo sobre una temática, artículo o política editorial más que una alabanza hacia el editor. La principal tarea de tanto el editor como del autor es la de comunicarse con el lector, no entre ellos. La bidireccionalidad entre autores y lectores es un proceso que facilita la difusión del conocimiento, somete a un nuevo juicio la información publicada lo que permite confrontar los hallazgos con una nueva realidad; asimismo permite generar nuevas interrogantes e ideas para investigar ${ }^{(26)}$. La interacción entre investigadores, académicos, lectores y estudiantes permite desarrollar a un campo de estudio, por lo que la redacción y publicación de cartas al editor es importante para la enseñanza y la transmisión del conocimiento científico.

La publicación de carta al editor y las respuestas entre autores y lectores favorece el factor de impacto de una revista, esto debido a que logra aumentar la cantidad de citas sobre un artículo que se está criticando y observando. De hecho, se conoce que con la finalidad de lograr un mayor factor de impacto las revistas suelen aceptar un gran número de cartas al editor, por lo que tienen una elevada tasa de aceptación ${ }^{(27)}$. La presente revisión encontró que la mayoría de cartas al editor se redactan para criticar un artículo; para corregir una declaración imprecisa, señalar errores o comentar la metodología. Como principal propósito se trata de iniciar y continuar un diálogo sustantivo, las cartas que simplemente están de acuerdo con todo lo que los autores han hecho e informado son poco probable que se publiquen.

Si bien las cartas al editor permiten interactuar a los lectores con los autores, estas publicaciones presentan polémicas pues algunos lectores las utilizan para criticar y ajustar cuentas personales con los autores, en algunas situaciones afectando la honra de investigadores; a veces se remiten cartas con nombres falsos para apoyar o 
criticar a un autor. Por otro lado, es bien sabido que las cartas al editor se aceptan más fácilmente que otros tipos de artículos científicos, por lo que pueden ser una puerta de entrada para que los autores sin méritos científicos ingresen sus nombres en revistas de alto factor de impacto y aumenten su producción científica ${ }^{(28)}$. Muchas veces estas cartas no son respondidas por los autores a quienes va dirigida, por lo que la esencia de la bidireccionalidad se pierde ${ }^{(29)}$. Aunque estos comentarios podrían haber llevado a algo importante, ignorarlos deja un vacío en el conocimiento de la investigación. Cabe indicar que una vez publicadas, estas cartas pueden citarse como publicaciones, citarse en otros artículos y utilizarse para respaldar solicitudes de subvenciones y empleo. Asimismo las cartas en revistas de renombre tienen el potencial de adquirir un respeto que tal vez no justifique.

\section{Conclusiones}

Una carta al editor es un tipo de publicación científica que se fundamenta en la interacción entre autor y lector. Su estructura y redacción es sencilla peo deben seguir un enfoque claro, y ofrecer críticas constructivas que se expresen con claridad. Esto permitirá una mayor probabilidad de que sea publicada por la revista. Se clasifican como cartas de comentario y cartas de observación. Las cartas de observación presentan una obra original, asemejan a un artículo original pero son más concisos. Las cartas del tipo "comentario" son las más comunes y constituyen una crítica a las publicaciones relacionadas con la revista. La finalidad de una carta radica en permitir expresar opiniones a favor o en contra de los hallazgos e ideas de los autores, desde un pisto de vista pedagógico al estudiante $y$ el investigador novel le permite introducirse en el campo de la redacción y comunicación científica. Estas publicaciones permiten el intercambio de opiniones entre autores y lectores, en algunas situaciones la carta al editor resulta igual de interesante que el articulo original que la originó. También permiten comunicar rápidamente hechos clínicos novedosos; por ejemplo cuando se reportó por primera vez los efectos adversos de la talidomida en embarazadas.

\section{Referencias}

1. Tang BL. Letter to the editor: On plurality and authorship in science. Account Res. 2018. [Acceso: 04/07/2020]; 25(4): 254-258. Disponible en: https: //pubmed.ncbi.nlm.nih.gov/29587536/

2. Anthony R. From the Editor: What's a letter to the editor? J Occup Environ Hyg. 2019. [Acceso: 04/07/2020]; 16(1): D1-D2. Disponible en: https: //pubmed.ncbi.nlm.nih.gov/30856088/

3. Falavarjani KG, Kashkouli MB, Chams H. Letter to Editor, a scientific forum for discussion. J Curr Ophthalmol. 2016. [Acceso: 04/07/2020]; 28(1): 1-2. Disponible en: https: //pubmed.ncbi.nlm.nih. gov/27239593/

4. Süer E, Yaman Ö. How to write an editorial letter? Turkish J Urol. 2013. [Acceso: 04/07/2020]; 39(Suppl 1): 41-3. Disponible en: https: //www.turkishjournalofurology.com/en/how-to-write-aneditorial-letter-13435

5. Dkhar SA. Letter to editor: its importance and drawbacks. Int J Community Med Public Health. 2018. [Acceso: 04/07/2020]; 5: 4634-6. Disponible en: https: //www.semanticscholar.org/paper/Letter-toeditor\%3A-its-importance-and-drawbacks-Dkhar/f6c43031714b842a84f785c912e83a5d20d0d4d6

6. International Committee of Medical Journal Editors. Uniform requirements for manuscripts submitted to biomedical journals: publishing and editorial issues related to publication in biomedical journals: correspondence. [Acceso: 04/07/2020]. Disponible en: www.icmje.org/ publishing_5correspond.html. 
7. Jadhav S, Bavdekar SB. Letter to Editor: Keeping the Dialogue Going. J Assoc Physicians India. 2015. [Acceso: 05/08/2020]; 63(5): 55-57. Disponible en: https: //pubmed.ncbi.nlm.nih.gov/26591146/

8. Anstey A. Letters to the editor: time for more scholarly debate. Br J Dermatol. 2014. [Acceso: 05/08/2020]; 171(1): 1-2. Disponible en: https: //onlinelibrary.wiley.com/doi/full/10.1111/bjd.13134

9. Why Write a Letter to the Editor?. Adv Neonatal Care. 2017. [Acceso: 05/08/2020]; 17(2): 77-78. Disponible en: https: //pubmed.ncbi.nlm.nih.gov/28362696/

10. López-Hernández D; Brito-Aranda L, Torres-Fonseca, A. Importancia y redacción de la carta al editor. Revista de Especialidades Médico Quirúrgicas. [Acceso: 05/08/2020]; 19(4): 475-478. Disponible en: https: //www.medigraphic.com/cgi-bin/new/resumen.cgi?IDARTICULO $=54588$

11. Sosa-Gonzalo SL, Aveiro-Róbalo TR, Galán-Rodas E. Cartas al editor: Importancia y recomendaciones para su redacción. CIMEL. 2016. [Acceso: 05/08/2020]; 21(2): 48- 50. Disponible en: https: //www. cimel.felsocem.net/index.php/CIMEL/article/view/643/361

12. Tierney E, O'Rourke C, Fenton JE. What is the role of 'the letter to the editor'? Eur Arch Otorhinolaryngol. 2015. [Acceso: 05/08/2020]; 272: 2089. Disponible en: https: //pubmed.ncbi.nlm.nih. gov/25231709/

13. Montenegro-Idrogo JJ, Mejía-Dolores JW, Chalco-Huamán JL. Cartas al editor publicadas en revistas biomédicas peruanas indizadas en SciELO- Perú 2006-2013. Rev Peru Med Exp Salud Publica. 2015. [Acceso: 05/08/2020]; 32(1): 104-9. Disponible en: http: //www.scielo.org.pe/scielo.php?script=sci arttext\&pid=S1726-46342015000100016

14. Revista de Investigación Clínica. Instrucciones a los autores. [Acceso: 05/08/2020]. Disponible en: http: //www.scielo.org.mx/revistas/ric/einstruc.htm

15. Fernández-Esteve, García AM. Sr. Director: La importancia de las Cartas al Director. Gac Sanit. 2005. [Acceso: 05/08/2020]; 19(5): 354-355. Disponible en: http: //scielo.isciii.es/scielo.php?script=sci_artt ext\&pid=S0213-91112005000500002

16. Miyahira JM. Importancia de las Cartas al editor. Rev Med Hered. 2010. [Acceso: 08/08/2020]; 21: 57-58.Disponible en: http: //www.scielo.org.pe/scielo.php?script=sci arttext\&pid=S1018130X2010000200001

17. Peh WC, Ng KH. Writing a letter to the Editor. Singapore Med J. 2010. [Acceso: 08/08/2020]; 51(7): 532-5. Disponible en: https: //pubmed.ncbi.nlm.nih.gov/20730391/

18. Gokani SA, Sharma E, Sharma T, Moudhgalya SV, Selvendran SS, Aggarwal N. Impact of a National Journal Club and Letter Writing Session on Improving Medical Students' Confidence with Critical Appraisal. Adv Med Educ Pract. 2019. [Acceso: 08/08/2020]; 10: 1081-1087. Disponible en: https: // pubmed.ncbi.nlm.nih.gov/31920419/

19. Miyahira JM. La investigación formativa y la formación para la investigación en el pregrado. Rev Med Hered. 2009. [Acceso: 08/08/2020]; 20(3): 119-122. Disponible en: https: //www.researchgate.net/ publication/262442184 La investigacion formativa y la formacion para la investigacion en el pregrado

20. Castro-Rodríguez Y. La lectura de artículos científicos. Entrevista a estudiantes del pregrado. Odontol. Sanmarquina. 2020. [Acceso: 08/08/2020]; 23(1): 93-95. Disponible en: https: //revistasinvestigacion. unmsm.edu.pe/index.php/odont/article/view/17516

21. Caballero-Ortiz AG. Cartas al editor como oportunidad de primera publicación en los miembros de FELSOCEM. CIMEL. 2015. [Acceso: 08/08/2020]; 19(2). Disponible en: http: //www.cimel.felsocem.net/index.php/CIMEL/article/viewArticle/494

22. Spodick DH, Goldberg RJ. The editor's correspondence: analysis of patterns appearing in selected specialty and general journals. Am J Cardiol. 1983 [Acceso: 20/02/2021]; 52: 1290- 1292. Disponible en: https: //pubmed.ncbi.nlm.nih.gov/6650419/

23. Johnson C, Green B. How to write a letter to the editor: an author's guide. J Chiropr Med. 2006. [Acceso: 08/08/2020]; 5(4): 144-7. Disponible en: https: //www.ncbi.nlm.nih.gov/pmc/articles/ PMC2647072/ 
24. Kearney MH. Write to Me Please: The Scholarly Importance of Letters to the Editor. Res Nurs Health. 2015. [Acceso: 08/08/2020]; 38(5): 327-9. Disponible en: https: //pubmed.ncbi.nlm.nih. gov/26291520/

25. Sturpe D, Kolar C, Janke K. Enhancing scholarly dialogue: Inviting Letters to the Editor. Curr Pharm Teach Learn. 2016. [Acceso: 08/08/2020]; 8(3): 269-70. Disponible en: https: //pubmed.ncbi.nlm. nih.gov/30070234l

26. Clouet D. Cartas al Editor: Algo más que 1.000 palabras. Rev Med Chile. 2014. [Acceso: 08/08/2020]; 142: 677-678. Disponible en: https: //scielo.conicyt.cl/scielo.php?script=sci arttext\&pi $\mathrm{d}=$ S0034-98872014000500021

27. Brown CJ. Letters to the editor provide a forum for readers and help make a journal accountable to the medical fraternity. Can Med Assoc J. 1997; 157: 792-4.

28. Moffatt B. Scientific authorship, pluralism, and practice. Account Res. 2018. [Acceso: 08/08/2020]; 25(4): 199-211. Disponible en: https: //pubmed.ncbi.nlm.nih.gov/29400074/

29. Neghina R, Neghina AM. How to build a scientific publishing career based on hundreds of letters-tothe-editor: -The Art of Lossll. Account Res. 2011. [Acceso: 08/08/2020]; 18(4): 247-9. Disponible en: https: //www.unboundmedicine.com/medline/citation/21707416/How_to_build_a_scientific_ publishing_career_based_on_hundreds_of_letters_to_the_editor:_\%22The_Art_of_Loss\%22_

\section{Nota conflicto de interés:}

El autor declara no tener conflictos de interés.

\section{Nota fuente de financiamiento:}

Autofinanciado.

\section{Nota contribución de los autores:}

1. Concepción y diseño del estudio

2. Adquisición de datos

3. Análisis de datos

4. Discusión de los resultados

5. Redacción del manuscrito

6. Aprobación de la versión final del manuscrito

YC ha contribuido en: $1,2,3,4,5$ y 6 .

\section{Nota de aceptación:}

Este artículo fue aprobado por la editora de la revista Mag. Dra. Vanesa Pereira-Prado. 